\title{
Seroepidemiology of Parechovirus A3 Neutralizing Antibodies, Australia, the Netherlands, and United States
}

\author{
Eveliina Karelehto, Lieke Brouwer, \\ Kimberley Benschop, Jen Kok, Kerri Basile, \\ Brendan McMullan, William Rawlinson, \\ Julian Druce, Suellen Nicholson, \\ Rangaraj Selvarangan, Christopher Harrison, \\ Kamani Lankachandra, Hetty van Eijk, \\ Gerrit Koen, Menno de Jong, \\ Dasja Pajkrt, Katja C. Wolthers
}

Recent parechovirus A3 (PeV-A3) outbreaks in Australia suggest lower population immunity compared with regions that have endemic PeV-A3 circulation. A serosurvey among populations in the Netherlands, the United States, and Australia before and after the 2013 Australia outbreak showed high PeV-A3 neutralizing antibody prevalence across all regions and time periods, indicating widespread circulation.

$\mathrm{P}$ arechovirus A3 (PeV-A3), belonging to the Picornavirus family, can cause respiratory and gastrointestinal symptoms, as well as meningitis and sepsis-like disease in infants (1). PeV-A3 was isolated from a fecal specimen collected in 1999 from a child with fever, diarrhea, and transient paralysis; it has been gaining increasing interest because of reported outbreaks of severe illness in neonates (2-4). To date the largest outbreaks have been caused by a recombinant PeV-A3 strain in Australia: in New South Wales in 2013, and in Victoria in 2015 (4). Humoral immunity is essential in protection against $\mathrm{PeV}-\mathrm{A} 3$ disease, yet seroepidemiological data on population immunity are

Author affiliations: Academic Medical Center, Amsterdam, the Netherlands (E. Karelehto, L. Brouwer, H. van Eijk, G. Koen, M. de Jong, D. Pajkrt, K.C. Wolthers); National Institute for Public Health and the Environment, Bilthoven, the Netherlands (K. Benschop); Institute of Clinical Pathology and Medical Research, Westmead, New South Wales, Australia (J. Kok, K. Basile); Sydney Children's Hospital, Sydney, New South Wales, Australia (B. McMullan); Prince of Wales Hospital, Randwick, New South Wales, Australia (W. Rawlinson); Doherty Institute, Melbourne, Victoria, Australia (J. Druce, S. Nicholson); Children's Mercy Hospital, Kansas City, Missouri, USA (R. Selvarangan,

C. Harrison); Truman Medical Center, Kansas City

(K. Lankachandra)

DOI: https://doi.org/10.3201/eid2501.180352 limited $(5,6)$. We describe the findings of a cross-sectional study on serum PeV-A3 neutralizing antibody (nAb) levels among children and adults from Victoria and New South Wales, Australia; Missouri, USA; and the Netherlands, where PeVs circulate every 2 years during summer and fall months $(3,7)$.

\section{The Study}

We screened 1,288 anonymized serum samples from persons $0-91$ years of age. From each geographic location, 2 independent sets of samples collected before and after the 2013 Australia PeV-A3 outbreak were used (Table 1). No ethics approval is required for anonymous use of biobank specimens in the Netherlands. Serum samples from the Netherlands in 2006-2007 came from a serum bank approved by the Medical Ethics Testing Committee of the Foundation of Therapeutic Evaluation of Medicines (ISRCTN 20164309). The institutional review board at the Children's Mercy Hospital (Kansas City, Missouri, USA) determined that anonymous use of the Missouri samples was exempt from ethics approval. The human research ethics committee at Melbourne Health approved the use of Victoria serum samples and the human research ethics committee at Western Sydney Local Health District approved the use of New South Wales serum samples (LNR/17/WMEAD/279).

We tested the serum samples with a previously described neutralization assay (8). We serially diluted heatinactivated serum samples and incubated them with chloroform-treated PeV-A3 strain isolated during the 2013 outbreak in Australia (GenBank accession no. KY930881) (4). We subsequently added LLCMK2 cells and incubated them for 7 days. We calculated neutralizing titers based on cytopathogenic effect using the Reed and Muench method and reported them as the reciprocal titers of serum dilutions exhibiting 50\% neutralization (9). We considered an $\mathrm{nAb}$ titer of $\geq 1: 8$ to be positive; we used $\geq 1: 32$ as a secondary cutoff (5). We compared PeV-A3 nAb seroprevalence between the timepoints within each location using $\chi^{2}$ tests. We performed logistic regression to examine the association between seropositivity and location-timepoint ( 8 categories), gender ( 2 categories), and age (3 categories). We present 3 univariable models and 1 multivariable model including all 3 variables. We used the Kruskal-Wallis test with post hoc analysis and Bonferroni correction to 
Seroepidemiology of Parechovirus A3

Table 1. Demographic information for study of parechovirus A3 neutralizing antibodies, Australia, the Netherlands, and United States*

\begin{tabular}{|c|c|c|c|c|c|}
\hline \multirow[b]{2}{*}{ Sample group } & \multirow[b]{2}{*}{ Institute } & \multirow[b]{2}{*}{ Sample type } & \multirow[b]{2}{*}{ No. (\%) patients } & \multicolumn{2}{|c|}{ Patient age, $y$} \\
\hline & & & & Mean & SD \\
\hline \multicolumn{6}{|l|}{ Country (state) and years } \\
\hline NL 2006-2007 & RIVM & $\mathrm{P}$ & $140(11)$ & 27.8 & 21.9 \\
\hline NL 2015-2016 & AMC & $\mathrm{R}, \mathrm{S}$ & $140(11)$ & 27.8 & 21.5 \\
\hline USA (MO) 2012-2013 & $\mathrm{CMH}$ & $\mathrm{R}$ & $120(9)$ & 30.8 & 18.3 \\
\hline USA (MO) 2017 & $\mathrm{CMH}, \mathrm{TMC}$ & $\mathrm{R}$ & 171 (13) & 25.5 & 18.8 \\
\hline AUS (VIC) 2011-2012 & VIDRL & $\mathrm{R}$ & $138(11)$ & 26.5 & 19.9 \\
\hline AUS (VIC) 2015-2016 & VIDRL & $\mathrm{R}$ & $138(11)$ & 26.4 & 19.6 \\
\hline AUS (NSW) 2011-2012 & WH, POW & $\mathrm{R}$ & $185(14)$ & 26.1 & 23.2 \\
\hline AUS (NSW) 2015-2016 & WH, POW & $\mathrm{R}$ & $257(20)$ & 23.9 & 20.6 \\
\hline \multicolumn{6}{|l|}{ Sext } \\
\hline M & & & $598(46)$ & 25.7 & 21.3 \\
\hline $\mathrm{F}$ & & & $580(45)$ & 25.5 & 20.5 \\
\hline \multicolumn{6}{|l|}{ Age, y } \\
\hline$<1$ & & & $148(11)$ & 0.4 & 0.3 \\
\hline $1-2$ & & & $52(4)$ & 1.8 & 0.6 \\
\hline $3-4$ & & & $41(3)$ & 3.8 & 0.6 \\
\hline $5-9$ & & & $120(9)$ & 7.2 & 1.5 \\
\hline $10-19$ & & & 220 (17) & 15.8 & 2.7 \\
\hline $20-29$ & & & $184(14)$ & 24.8 & 2.9 \\
\hline 30-39 & & & $172(13)$ & 34.2 & 2.8 \\
\hline $40-49$ & & & $162(13)$ & 44.6 & 2.8 \\
\hline $50-59$ & & & $89(7)$ & 54.8 & 3.2 \\
\hline $60-69$ & & & $62(5)$ & 64.3 & 2.8 \\
\hline$>69$ & & & $38(3)$ & 76.4 & 5.3 \\
\hline Total & & & 1,288 & & \\
\hline \multicolumn{6}{|c|}{$\begin{array}{l}\text { “AMC, Academic Medical Center; AUS, Australia; CMH, Children's Mercy Hospital; NL, the Netherlands; P, population-based sampling; POW, Prince of } \\
\text { Wales Hospital; R, residual serum from hospitalized patients and community; RIVM, National Institute for Public Health and the Environment; S, AMC } \\
\text { staff; SD, standard deviation; TMC, Truman Medical Center; VIC, Victoria; NSW, New South Wales; VIDRL, Victorian Infectious Diseases Reference } \\
\text { Laboratory; WH, Westmead Hospital. } \\
\text { †Information on sex not available for US } 2017 \text { adult samples. }\end{array}$} \\
\hline
\end{tabular}

compare the median $\mathrm{nAb}$ titers. In the statistical analyses, we excluded children $<1$ year of age because of the presence of maternal antibodies; we merged the remaining age categories into 3 groups.

Overall PeV-A3 nAb seropositivity was similar across 3 locations: $71.1 \%(2006-2007)$ and $69.2 \%(2015-2016)$ in the Netherlands, $63.3 \%(2012-2013)$ and $66.5 \%$ (2017) in Missouri, and 58.5\% (2011-2012) and 66.4\% (2015-2016) in Victoria (Figure 1, panel A). In New South Wales, nAb seroprevalence was $82.9 \%$ in $2011-2012$, whereas it was significantly less $(68.6 \%)$ in $2015-2016(\mathrm{p}=0.005$; Figure 1 , panel A).

Age was a significant determinant of PeV-A3 nAb seropositivity, which increased from $32.7 \%$ in children $1-2$ years of age to $65.0 \%$ in those 5-9 years of age and peaked at $77.7 \%$ in adults $20-30$ years of age (Figure 1, panel B). $\mathrm{nAb}$ seropositivity decreased to $42.1 \%$ in persons $>30$ years of age when a titer cutoff $\geq 1: 32$, the level necessary for protection against disease (5), was used. Furthermore, we observed that only $33.8 \%$ of infants $<1$ year of age had an $\mathrm{nAb}$ titer $\geq 1: 32$ and were thus sufficiently protected by maternal antibodies (Figure 1, panel B).

We compiled age-stratified seroprevalences for each location and timepoint (Figure 1, panels $\mathrm{C}-\mathrm{F}$ ). The variables location-timepoint and age were significantly associated with seroprevalence in both univariable and multivariable regression models $(\mathrm{p}<0.002$; Table 2$)$. We did not detect sex-dependent differences $(p=0.309$; Table 2). In line with the age-stratified seropositivity, the geometric mean titers (GMTs) declined steadily with age (Figure 2). Overall GMT peaked at 1:53 (SD 8.5) in the 10-19year age group and decreased thereafter. Both children $1-5$ years of age $(p=0.001)$ and adults $>30$ years of age $(p<0.001)$ had significantly lower median titers than persons 6-29 years of age.

\section{Conclusions}

In this large seroepidemiological PeV-A3 study, we compared the $\mathrm{nAb}$ prevalence in populations from 4 distinct geographic regions. We report high and comparable PeVA3 nAb seropositivity across all these regions. In agreement with the reports from Japan, the overall seroprevalence was $68.9 \%$, suggesting widespread global circulation of PeV-A3 $(10,11)$. Unexpectedly, the level of PeV-A3 humoral immunity in NSW was higher before the 2013 outbreak compared with 2-3 years after the outbreak. This suggests that PeV-A3 was already endemic in Australia before or during 2011-2012. Localized smaller PeV-A3 upsurges or variations in the proportion of samples originating from hospitalized patients versus the community may explain the observed difference between the earlier and later time periods.

Age-stratified PeV-A3 nAb seropositivity and GMTs suggest that the infection generally occurs in children $<10$ 

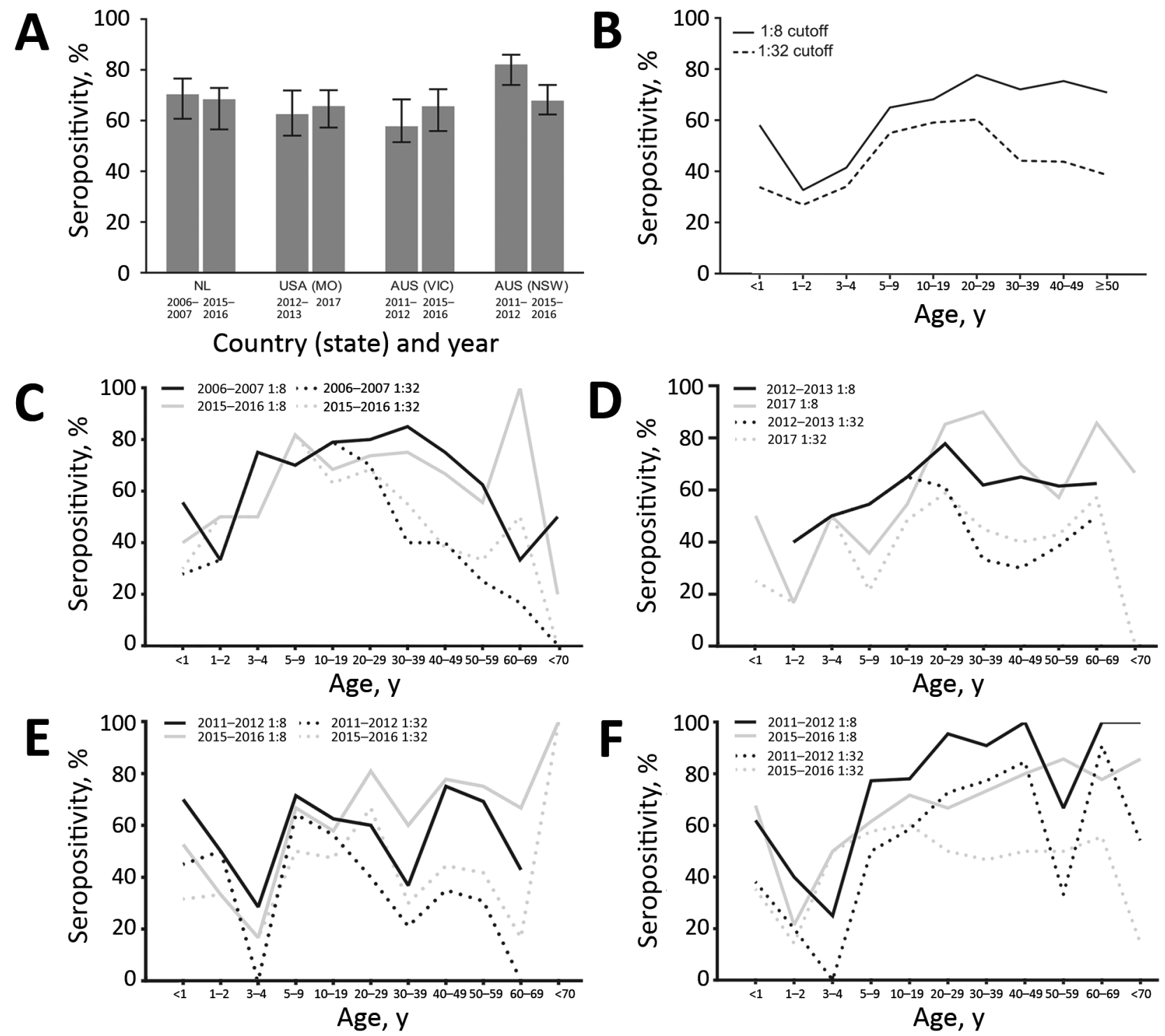

Figure 1. Parechovirus A3 (PeV-A3) neutralizing antibody ( $\mathrm{AAb}$ ) seropositivity, Australia, the Netherlands, and United States. A) Overall nAb seropositivity with associated $95 \% \mathrm{Cls}$. Infants $<1$ year of age were excluded from the analysis. Seropositivity rates between the timepoints within each location were compared by using $\chi^{2}$ tests. B) Overall age-stratified PeV-A3 nAb seropositivity, including infants $<1$ year of age. Seropositivity was determined as a nAb titer of $\geq 1: 8$ or $\geq 1: 32$. C-F) Age-stratified PeV-A3 nAb seropositivity in C) the Netherlands;

D) Missouri, USA; E) Victoria, Australia; and F) New South Wales, Australia. Complete data used in this figure can be found in the Appendix (http://wwwnc.cdc.gov/EID/article/25/1/18-0352-App1.pdf). AUS, Australia; NL, the Netherlands; NSW, New South Wales; VIC, Victoria.

years of age, although nAb titers continued to increase in adolescent children. $\mathrm{nAb}$ titers decreased below the proposed level of protection in adults $>30$ years of age. Similar observations have been reported previously $(5,10,12)$. This result is in contrast to results for PeV-A1, against which high $\mathrm{nAb}$ seropositivity rates are maintained in adults (11). The large proportion of seronegative persons and gradually declining GMTs in older age categories may indicate that widespread circulation of PeV-A3 has emerged fairly recently, as previously proposed (13), or that the immunity elicited in childhood is waning. Because the mean age of women at first birth in developed countries is high, we hypothesize that low nAb titers in women of childbearing age, and therefore the lack of adequate maternal antibody protection, contribute to the occurrence of PeV-A3 outbreaks in infants. Moreover, the 2013 Australia outbreak strain was recently described as a novel recombinant with the capsid-encoding region of the genome originating from a PeV-A3 strain collected in Japan in 2011 and the nonstructural region from an unknown origin (4). Preexisting serum antibodies recognizing epitopes in the $\mathrm{PeV}$ A3 capsid maintain their ability to neutralize this strain, but this factor may represent a more virulent variant of PeV-A3. 
Table 2. Association between seropositivity and location-timepoint, gender, and age by univariate and multivariate logistic regression models in study of parechovirus A3 neutralizing antibodies, Australia, the Netherlands, and United States*

\begin{tabular}{|c|c|c|c|c|}
\hline \multirow[b]{2}{*}{ Parameter } & \multicolumn{2}{|c|}{ Univariate model } & \multicolumn{2}{|c|}{ Multivariate model } \\
\hline & Odds ratio $(95 \% \mathrm{Cl})$ & $\mathrm{p}$ value & Odds ratio $(95 \% \mathrm{Cl})$ & $\mathrm{p}$ value \\
\hline Location, years & & 0.002 & & $<0.001$ \\
\hline Netherlands, 2006-2007 & $1.124(0.693-1.824)$ & & $1.078(0.658-1.764)$ & \\
\hline Netherlands, 2015-2016 & $1.026(0.635-1.658)$ & & $0.963(0.591-1.568)$ & \\
\hline Missouri, USA, 2011-2012 & $0.790(0.496-1.260)$ & & $0.725(0.451-1.166)$ & \\
\hline Missouri, USA, 2017 & $0.906(0.585-1.403)$ & & $0.376(0.189-0.748)$ & \\
\hline Victoria, Australia, 2011-2012 & $0.644(0.406-1.023)$ & & $0.642(0.400-1.030)$ & \\
\hline Victoria, Australia, 2015-2016 & $0.904(0.563-1.451)$ & & $0.868(0.536-1.406)$ & \\
\hline New South Wales, Australia, 2011-2012 & $2.222(1.354-3.647)$ & & $2.247(1.358-3.718)$ & \\
\hline New South Wales, Australia, 2015-2016† & 1 & & 1 & \\
\hline Sexł & & 0.193 & & 0.309 \\
\hline $\mathrm{F}$ & $0.840(0.646-1.092)$ & & $0.868(0.661-1.140)$ & \\
\hline $\mathrm{M} \dagger$ & 1 & & 1 & \\
\hline Age, y & & $<0.001$ & & $<0.001$ \\
\hline $1-5 \dagger$ & 1 & & 1 & \\
\hline $6-29$ & $2.938(1.954-4.419)$ & & $2.782(1.821-4.251)$ & \\
\hline$\geq 30$ & $3.248(2.160-4.883)$ & & $3.081(2.004-4.738)$ & \\
\hline
\end{tabular}

*Boldface indicates a statistically significant result. Seropositivity was determined as a neutralizing antibody titer $\geq 1: 8$. Children $<1$ years of age were excluded.

tReference category.

łInformation on sex not available for US 2017 adult samples.

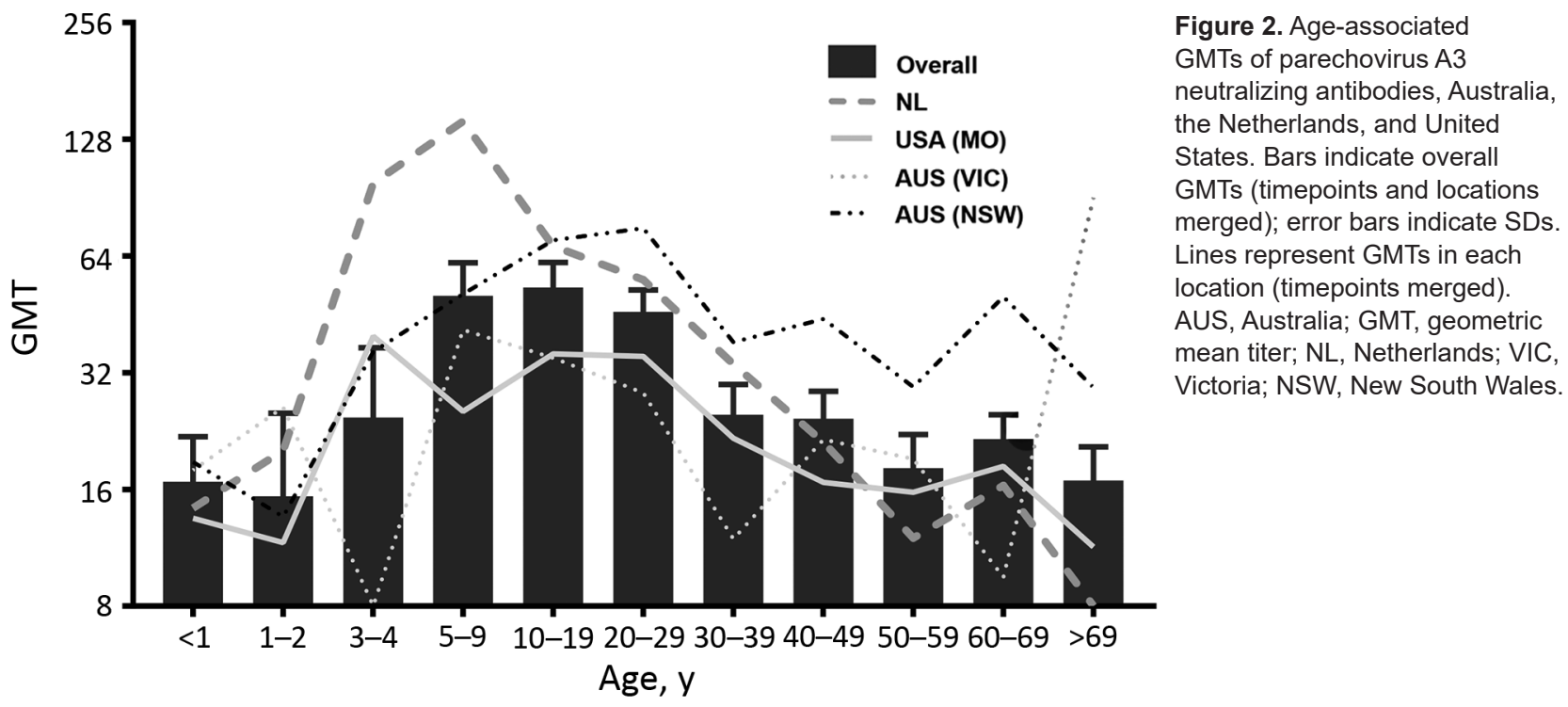

This study has limitations. Cross-neutralizing antibodies resulting from exposure to other PeV genotypes may confound our findings. However, we have previously observed no evidence of PeV-A3 cross-neutralization by polyclonal and monoclonal antibodies elicited against PeV-A1 to $5(14,15)$. Because we used anonymous serum samples from population-based sampling and residual serum collections, we could not relate the seroprevalence to cohort exposure history or etiologic information, and the varying sampling time periods prohibit us from making direct temporal comparisons between the locations.

Taken together, our results suggest that PeV-A3 circulation is widespread and that infection takes place in early childhood and adolescence. Nonetheless, PeV-A3 outbreaks occur regularly in young infants, and case numbers remain elevated in Australia (L. Caly, Doherty Institute, Melbourne, VIC, Australia, pers. comm. 2017 Oct 15). Why humoral immunity against $\mathrm{PeV}-\mathrm{A} 3$ declines with age and what factors predispose neonates to severe PeV-A3 illness remain to be elucidated. Implementation of molecular $\mathrm{PeV}$ detection in routine diagnostics and continuous surveillance are warranted.

\section{Acknowledgments}

We thank Laura Teixidó for excellent technical assistance and Rebecca Holman for expert advice on statistical analyses.

This research was funded by the FP7 Marie Curie Industry Academia Partnerships and Pathways consortium Academia Industry R\&D Opportunities for Picornaviruses. 


\section{About the Author}

Ms. Karelehto is a PhD candidate at the Laboratory of Clinical Virology in the Department of Medical Microbiology at the Academic Medical Center in Amsterdam, the Netherlands. Her research focuses on parechovirus seroepidemiology and virus-host interactions.

\section{References}

1. Harvala H, Wolthers KC, Simmonds P. Parechoviruses in children: understanding a new infection. Curr Opin Infect Dis. 2010; 23:224-30. http://dx.doi.org/10.1097/QCO.0b013e32833890ca

2. Ito M, Yamashita T, Tsuzuki H, Takeda N, Sakae K. Isolation and identification of a novel human parechovirus. J Gen Virol. 2004;85:391-8. http://dx.doi.org/10.1099/vir.0.19456-0

3. Midgley CM, Jackson MA, Selvarangan R, Franklin P, Holzschuh EL, Lloyd J, et al. Severe parechovirus 3 infections in young infants - Kansas and Missouri, 2014. J Pediatric Infect Dis Soc. 2018;7:104-12. http://dx.doi.org/10.1093/jpids/pi

4. Nelson TM, Vuillermin P, Hodge J, Druce J, Williams DT, Jasrotia R, et al. An outbreak of severe infections among Australian infants caused by a novel recombinant strain of human parechovirus type 3. Sci Rep. 2017;7:44423. http://dx.doi.org/10.1038/srep44423

5. Aizawa Y, Watanabe K, Oishi T, Hirano H, Hasegawa I, Saitoh A. Role of maternal antibodies in infants with severe diseases related to human parechovirus type 3. Emerg Infect Dis. 2015;21:1966-72. http://dx.doi.org/10.3201/eid2111.150267

6. Westerhuis B, Kolehmainen P, Benschop K, Nurminen N, Koen G, Koskiniemi M, et al. Human parechovirus seroprevalence in Finland and the Netherlands. J Clin Virol. 2013;58:211-5. http://dx.doi.org/10.1016/j.jcv.2013.06.036

7. Janes VA, Minnaar R, Koen G, van Eijk H, Dijkman-de Haan K, Pajkrt D, et al. Presence of human non-polio enterovirus and parechovirus genotypes in an Amsterdam hospital in 2007 to 2011 compared to national and international published surveillance data: a comprehensive review. Euro Surveill. 2014;19:20964. http://dx.doi.org/10.2807/1560-7917.ES2014.19.46.20964
8. Karelehto E, van der Sanden S, Geraets JA, Domanska A, van der Linden L, Hoogendoorn D, et al. Strain-dependent neutralization reveals antigenic variation of human parechovirus 3. Sci Rep. 2017;7:12075. http://dx.doi.org/10.1038/s41598-017-12458-5

9. Reed LJ, Muench H. A simple method of estimating fifty percent endpoints. Am J Hyg. 1938;27:493-7.

10. Watanabe K, Hirokawa C, Tazawa T. Seropositivity and epidemiology of human parechovirus types 1, 3, and 6 in Japan. Epidemiol Infect. 2016; (Aug):1-10.

11. Tanaka S, Aoki Y, Matoba Y, Yahagi K, Itagaki T, Matsuzaki Y, et al. Seroepidemiology of human parechovirus types 1, 3, and 6 in Yamagata, Japan, in 2014. Microbiol Immunol. 2016;60:854-8. http://dx.doi.org/10.1111/1348-0421.12456

12. Karelehto E, Wildenbeest J, Benschop K, Koen G, Rebers S, Bouma-de Jongh S, et al. Human parechovirus 1, -3 and -4 neutralizing antibodies in Dutch mothers and infants and their role in protection against disease. Pediatr Infect Dis J. http://dx.doi.org/10.1097/INF.0000000000001986

13. Calvert J, Chieochansin T, Benschop KS, McWilliam Leitch EC, Drexler JF, Grywna K, et al. Recombination dynamics of human parechoviruses: investigation of type-specific differences in frequency and epidemiological correlates. J Gen Virol. 2010;91: 1229-38. http://dx.doi.org/10.1099/vir.0.018747-0

14. Westerhuis BM, Koen G, Wildenbeest JG, Pajkrt D, de Jong MD, Benschop KS, et al. Specific cell tropism and neutralization of human parechovirus types 1 and 3: implications for pathogenesis and therapy development. J Gen Virol. 2012;93:2363-70. http://dx.doi.org/10.1099/vir.0.043323-0

15. Westerhuis BM, Benschop KS, Koen G, Claassen YB, Wagner K, Bakker AQ, et al. Human memory B cells producing potent cross-neutralizing antibodies against human parechovirus: implications for prevalence, treatment, and diagnosis. J Virol. 2015;89:7457-64. http://dx.doi.org/10.1128/JVI.01079-15

Address for correspondence: Katja C. Wolthers, Laboratory of Clinical Virology, Rm L1-109, Department of Medical Microbiology, Academic Medical Center, University of Amsterdam, Meibergdreef 9, 1105AZ

Amsterdam, the Netherlands; email: k.c.wolthers@amc.uva.nl
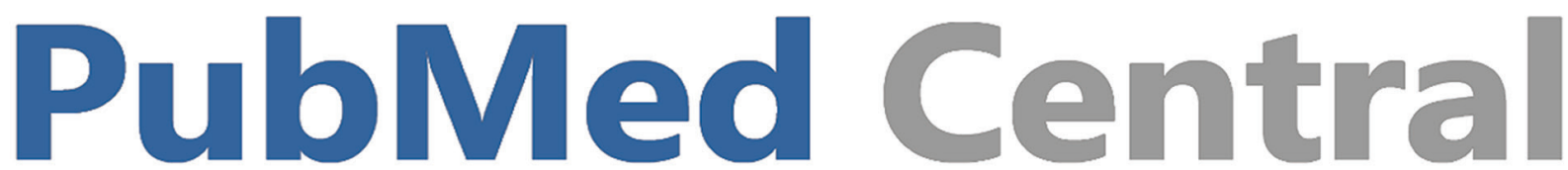

\title{
PublMed
}

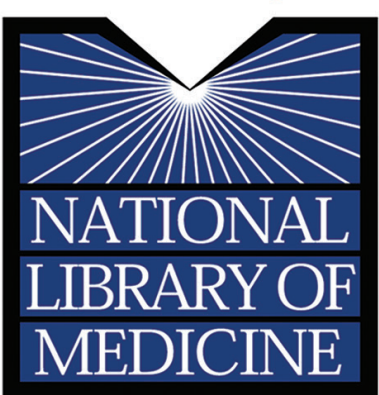

\section{Find Emerging Infectious Diseases content in the digital archives of the National Library of Medicine}

\author{
www.pubmedcentral.nih.gov
}

